(231)

\title{
Interdisciplinary Approach in Finding Integrated Management Solutions to Complex Issues: Case of CKDu in Sri Lanka
}

\author{
Ranasinghe M. \\ Faculty of Graduate Studies, York University, Canada \\ maxran1@yahoo.com
}

\begin{abstract}
The purpose of adopting "Interdisciplinary Approach" is to view the issue in different lenses, to identify the commonalities and integrate those, find gaps if any, in different disciplinary views and fill those gaps with the views found in other disciplines to expand the existing knowledge. This paper reports integrated management solutions found in a study using an interdisciplinary approach on the Chronic Kidney Disease of Unknown Etiology (CKDu). The Chronic Kidney Disease of Unknown Etiology (CKDu) was discovered by the Ministry of Health in the 1990s among the paddy farmers in the North Central Province (NCP) of Sri Lanka. The CKDu is a new form of chronic kidney disease (CKD). The Government Medical Officers Association of Sri Lanka (GMOAS) states that more than 40,000 people are affected countrywide (GMOAS, 2013). Since the problem is complex, interconnected to different domains, and many stakeholders are involved, no single disciplinary approach can provide a comprehensive answer to the problem of CKDu in Sri Lanka. Thus, an interdisciplinary approach has been chosen which looked at the issue from different viewpoints, i.e., environmental, social capitol (support), legal and corporate responsibility. The main objectives of the study were to explore and analyze the effectiveness of the processes and measures so far adopted by the Government of Sri Lanka to handle the issue; to identify the potential of the village level organizations and support groups in developing a solution to the CKDu issue; and to critically analyze the response of agro-chemical companies to the CKDu crisis in Sri Lanka, the legal environment in which they operate, and how to establish their responsibilities and accountability in the search for solutions to the CKDu crisis. Perspectives Taking Theory" is adopted as the conceptual framework throughout the study as a solid theoretical basis for the study. Secondary data were gathered from published literature and primary data were gathered using structured and unstructured interviews, key informant surveys, focal group discussions. All possible stakeholders were interviewed including Government officials, agrochemical companies, lawyers, environmentalists, researchers and affected and non-affected communities. For the community survey Padaviya Division was selected as has a very high prevalence for CKDu. According to the results, degradation of the environment, unplanned agriculture, blind embracing of industrial agriculture, negligence of and caring and restoring the cascade tank systems are major causes of CKDu. Despite efforts by the officials of the Ministry of Health in largely the treatment and to a lesser extent prevention of the disease the cause and effect of the disease had not been looked at in a wholesome manner. The Government has to take a wellcoordinated and monitored integrated approach with its multitude of agencies in providing a lasting solution to this issue in the short, medium and long terms. Creating awareness of the disease, early detection, strengthening the implementation of law as to use of agro-chemicals, ensure the supply of clean water are the immediate and short-term remedies but in creating long term remedies it is needed to change the attitudes of the farmers in the over use of agrochemicals or encourage and support them to resort to organic agriculture. As there is no concrete evidence that the agrochemicals are the direct culprit to the disease, it is not practical to sue them for damages. However, through the corporate social responsibility, they can be included in the equation which is more long lasting and sustainable. The existing village level social capital, including religious bodies, civil society organizations, and other resources can be mobilized effectively to address the CKDu problem at village level which is a resource hitherto untapped.
\end{abstract}

Keywords: CKDu, Interdisciplinary, Integrated, Agrochemicals, Corporate social responsibility

Proceedings of the International Forestry and Environment Symposium 2017 of the Department of Forestry and Environmental Science, University of Sri Jayewardenepura, Sri Lanka 